\title{
Validation of the Simplified Inguinal Pain Questionnaire for assessing postoperative pain and disability following hernioplasty
}

\author{
Jose L. Ramirez-GarciaLuna ${ }^{1,2,3}$ (1) - Jorge Aguilar-Garcia ${ }^{2,3} \cdot$ Rodrigo Fernandez-Villafuerte $^{3}$. \\ Mario A. Matinez-Jimenez ${ }^{2,3}$ (D)
}

Received: 25 November 2020 / Accepted: 2 January 2021 / Published online: 2 April 2021

(c) Springer Nature Singapore Pte Ltd. 2021

\begin{abstract}
The purpose of this study was to assess the psychometric properties of the Simplified Inguinal Pain Questionnaire (sIPQ) and compare it to the regular Inguinal Pain Questionnaire (IPQ) for external validation. To do so, the IPQ and sIPQ were telephonically administered at 7 and 28 days postoperatively for a cohort of 25 patients who underwent Lichtenstein hernioplasty. A psychometric analysis of the scores was done using Cronbach's alpha and test-retest assessments. The agreement rate of the mean-standardized values between scores was afterwards calculated. We found closely similar psychometric values for the IPQ and sIPQ. The agreement rate between scores was 97\% $(p<0.001)$. Taken together, the results demonstrate that the sIPQ has psychometric values that are very similar to those of the full IPQ. Its shorter number of items is specifically designed to enable telephonic follow-up and streamline both postoperative care and telemedicine.
\end{abstract}

Keywords Inguinal hernia $\cdot$ Postoperative inguinal pain $\cdot$ Validation studies

In a recent paper [1], using machine learning methods, our research team developed a simplified version of the Inguinal Pain Questionnaire (IPQ) by Fränneby et al. [2] This psychometric tool is designed to measure postoperative pain and disability following hernioplasty to identify those suffering from chronic pain. In the simplified version of the IPQ (sIPQ), we reduced the number of items from 18 to 8 . This reduction was specifically designed to decrease the time required for its completion and enable telephonic follow-up of postoperative patients abided by the ongoing COVID-19 pandemic. While the psychometric properties of the sIPQ were found to be almost identical to those of the original IPQ in its developing cohort, no validation of the score was done.

Jose L. Ramirez-GarciaLuna

jose.ramirezgarcialuna@mail.mcgill.ca

1 Division of Experimental Surgery, Faculty of Medicine, McGill University, 3605 Rue de la Montagne, Montréal, QC H3G 2M1, Canada

2 Department of Surgery, Faculty of Medicine, Universidad Autonoma de San Luis Potosi, 2405 Venustiano Carranza Ave, 78210 San Luis Potosi, SLP, Mexico

3 Division of General Surgery, Hospital Central "Dr. Ignacio Morones Prieto", 2395 Venustiano Carranza Ave, 78290 San Luis Potosi, SLP, Mexico
Therefore, in this study, we assess the sIPQ psychometric properties in an independent patient cohort.

Following Lichtenstein inguinal hernioplasty, 25 patients were enrolled in the study. Their characteristics are presented in Table 1. This sample size was calculated following the same steps and results of our previous study as well as the recommendation of a subject-to-item ratio $\geq 2[1,3]$. The IPQ and SIPQ were telephonically administered at 7 days and 28 days postoperatively. The order in which the scores were administered was randomized to avoid bias. The time required to complete the IPQ was 13 IQR $5 \mathrm{~min}$, compared to 7 IQR 3 min for the sIPQ $(p<0.001$ in a paired Wilcoxonrank sum test). Afterwards, we compared the full score values against those of the sIPQ (Table 2). Spearman's rank correlation between the full IPQ and the sIPQ was 0.792, $p<0.001$. Cronbach's alpha for the IPQ data was 0.70 (95\% CI 0.60-0.77), while for the sIPQ was 0.68 (95\% CI $0.58-0.80$ ). The score's temporal stability was assessed by calculating the Spearman's rank correlation of 7- and 28-day measurements. For the IPQ, its temporal stability was 0.68 (95\% CI $0.52-0.82, p<0.001)$ and for the sIPQ, 0.54 (95\% CI $0.41-0.71, p<0.001)$. Finally, to obtain the intraclass 
Table 1 Patient characteristics

\begin{tabular}{ll}
\hline Variable & $N=25$ \\
\hline Age & $46.7 \pm 13.5$ \\
Gender & Female $=5(20 \%)$ \\
& Male $=15(80 \%)$ \\
Hernia classification & NYHUS I $=6(24 \%)$ \\
& NYHUS II $=7(28 \%)$ \\
& NYHUS IIIa $=7(28 \%)$ \\
& NYHUS IIIb $=5(20 \%)$ \\
Inguinal pain questionnaire score & 7 -day PO $=22.3 \pm 8.8$ \\
& 28-day PO $=17.7 \pm 7.0$ \\
Simplified inguinal pain questionnaire score & 7 -day PO $=10.7 \pm 4.7$ \\
& 28-day PO $=8.6 \pm 3.5$ \\
\hline
\end{tabular}

Data are presented as the mean and standard deviation or proportions, as appropriate

correlation coefficient (ICC) between the scores, we standardized them by subtracting the mean score value from each individual value and dividing the resulting number by the standard deviation. In that way, pairwise mean-standardized value comparisons were performed. The type 3-2 ICC between scores was 0.97 (95\% CI 0.94-0.98) (Fig. 1).

Taken together, our results demonstrate that the sIPQ has psychometric properties that are almost identical to those of the full IPQ, thereby adequately capturing pain and disability following hernioplasty. While other abbreviated forms of the IPQ exist [4], the sIPQ was developed using a rigorous statistical analysis in contrast to heuristically determining which items would be more clinically relevant. Furthermore, the sIPQ was also developed from patient's data collected in the immediate and mediate postoperative period, rather than after several years after surgery. Some potential drawbacks of the SIPQ include the fact that it does not consider pre-operative pain (question 1 of the full IPQ), thus, failing to establish a pain baseline; and not considering other daily activities that may trigger pain and impact quality of life, except for sitting and standing. However, in our opinion, the most significant value that the sIPQ offers is enabling data collection in a consistent and abbreviated manner, thereby fostering its use for telemedicine. Consultations and surgical follow-up using virtual modalities during the COVID-19 pandemic has been found to be safe and effective and will likely be the norm for low-risk patients in the foreseeable future $[5,6]$. Using validated, abbreviated, and refined tools can, therefore, make the data collection more efficient, allow for better telemedicine assessments, and the timely and correct identification of patients who may need in-person consultations. 
Table 2 Simplified inguinal pain questionnaire

Item Scoring

Estimate the pain you feel right now in the groin on the same side as the operation

0-No pain

1-Pain present but can easily be ignored

2-Pain present, cannot be ignored, but does not interfere with everyday activities

3-Pain present, cannot be ignored, interferes with concentration on chores and daily activities

4-Pain present, cannot be ignored, interferes with most activities

5-Pain present, cannot be ignored, necessitates bed rest

6-Pain present, cannot be ignored, prompt medical advice sought

Estimate the worst pain you felt in the operated groin during this past week

0 - No pain

1-Pain present but can easily be ignored

2-Pain present, cannot be ignored, but does not interfere with everyday activities

3-Pain present, cannot be ignored, interferes with concentration on chores and daily activities

4-Pain present, cannot be ignored, interferes with most activities

5-Pain present, cannot be ignored, necessitates bed rest

6-Pain present, cannot be ignored, prompt medical advice sought

How often have you felt pain in the operated groin during the past week? 1 -Once a week

$2-2$ to 5 times a week

3-Every day

4-Every day and also during nighttime

5-I have had pain the whole week, both at day and night

Do you find it difficult sitting down for more than half an hour because of $0-$ No

the pain?

1 -Yes

Do you find it difficult standing up for more than half an hour because of $0-$ No

the pain?

$1-$ Yes

Have you on any occasion taken painkillers for pain in the operated groin? $0-$ No

1 -Yes

Estimate the severity of pain you feel right now in the groin opposite to

0 - No pain the operated side

1-Pain present but can easily be ignored

2-Pain present, cannot be ignored, but does not interfere with everyday activities

3-Pain present, cannot be ignored, interferes with concentration on chores and daily activities

4-Pain present, cannot be ignored, interferes with most activities

5-Pain present, cannot be ignored, necessitates bed rest

6-Pain present, cannot be ignored, prompt medical advice sought

Estimate the worst pain you have felt in the groin opposite to the operated side during this past week

0 - No pain

1-Pain present but can easily be ignored

2-Pain present, cannot be ignored, but does not interfere with everyday activities

3-Pain present, cannot be ignored, interferes with concentration on chores and daily activities

4-Pain present, cannot be ignored, interferes with most activities

5-Pain present, cannot be ignored, necessitates bed rest

6-Pain present, cannot be ignored, prompt medical advice sought 
A)

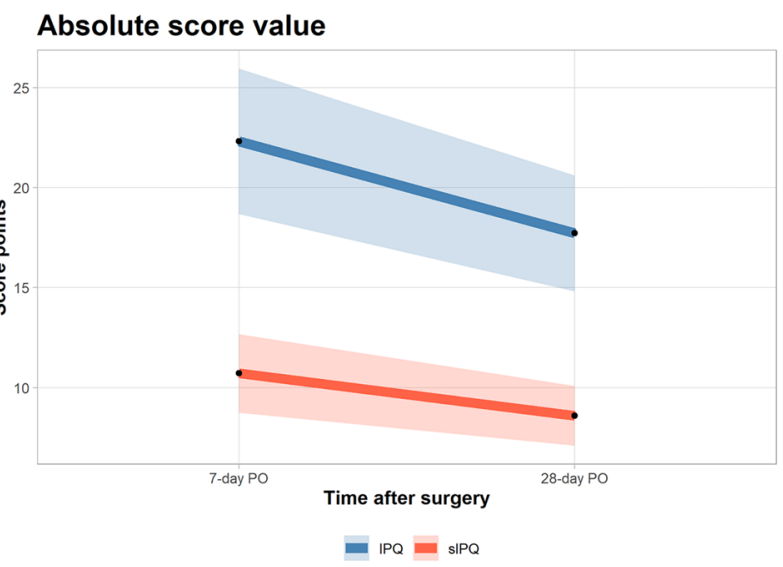

C)

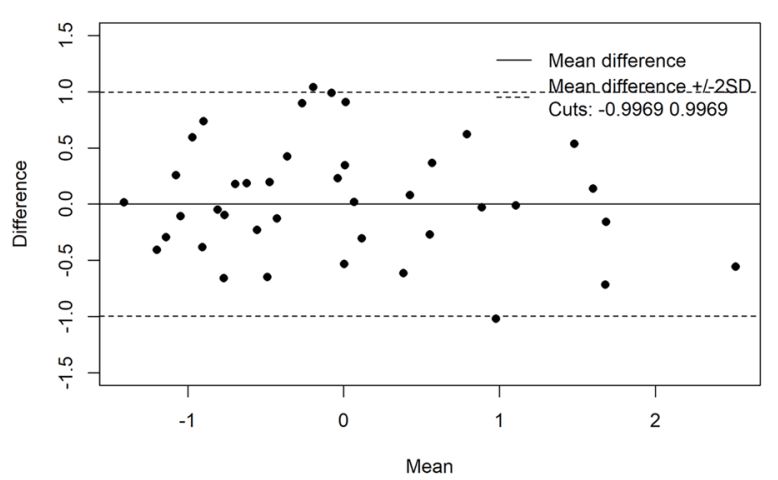

Fig. 1 Psychometric evaluation of the scores. The full value of the Inguinal Pain Questionnaire (IPQ) and its simplified version (sIPQ) were compared. Although there was a significant difference in their absolute values, the change slope between 7- and 28-day measurements is similar for the IPQ (a, blue) and the sIPQ (a, red). The cor-

Acknowledgements MAMJ receives doctoral support from the Mexican Council of Science and Technology (CONACYT). JLRG receives postdoctoral support from a Mitacs Elevate Fellowship.

Author contributions Conceptualization: JLRGL and MAMJ. Methodology: JLRGLand JAG. Data acquisition and curation: RVF and JAG. Statistical analysis: JLRGL. Supervision: JLRGL and MAMJ. Manuscript writing, review and editing: All authors.

Funding No specific funding was received for this project. All authors declare no conflicts of interest. MAMJ receives doctoral support from the Mexican Council of Science and Technology (CONACYT). JLRG holds a Mitacs Elevate Postdoctoral Fellowship. The study was approved by Hospital Central "Dr. Ignacio Morones Prieto" Research Ethics Board, as well as by the State of San Luis Potosi Ministry of Health (Registry 48-18 and CONBIOETICA-24CEI-001-20160427, respectively). All study subjects provided informed consent for participation in the study. The study's data are available upon request to the authors.

\section{Declarations}

Conflict of interest JLRG declares that he has no conflict of interest. JAG declares that he has no conflict of interest. RVF declares that he
B)

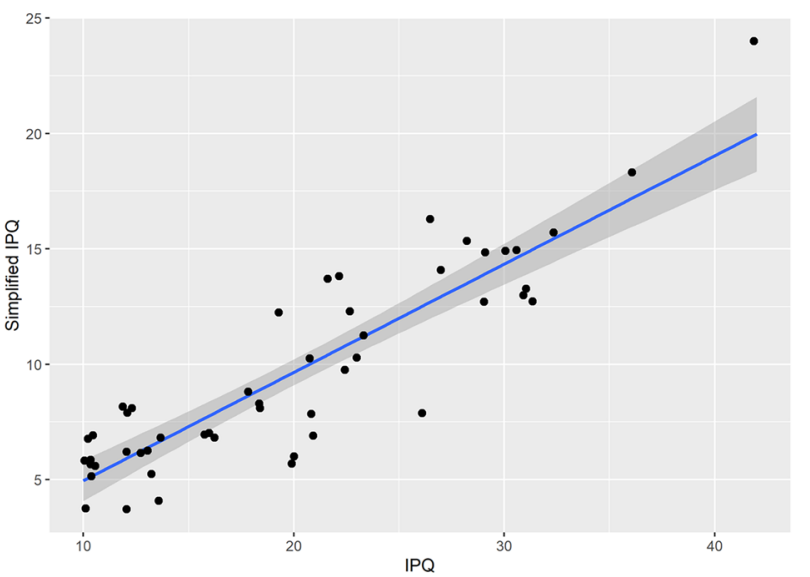

D)

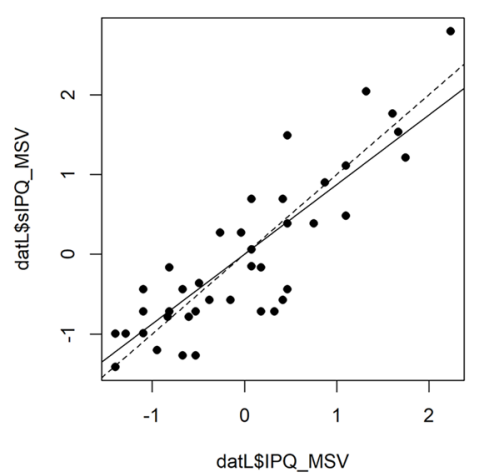

relation between the score absolute values was $79.2 \%(p<0.001)(\mathbf{b})$, while the intraclass correlation coefficient for their mean-standardized values was $97.0 \%(p<0.001)$ as shown in a Bland-Altman plot (c) and an expected vs. observed values plot (d). The shaded areas in the figure panels represent $95 \%$ CI

has no conflict of interest. MAMJ declares that he has no conflict of interest.

Ethical approval All procedures performed in the studies involving human participants were in accordance with the ethical standards of the institutional and national research committee (Registry 48-18 and CONBIOETICA-24CEI-001-20160427) and with the 1964 Helsinki Declaration and its later amendments.

Informed consent Informed consent was obtained from all individual participants included in the study.

\section{References}

1. Aguilar-García J, Villafuerte-Fernandez R, Ntezes-Hidalgo PI, Meade-Aguilar JA, Ramirez-GarciaLuna JL, Martinez-Jimenez MA. Postoperative inguinal pain and disability after Lichtenstein versus ONSTEP hernia repair: analysis of responses to the inguinal pain questionnaire in Spanish. Surg Today. 2020. https://doi. org/10.1007/s00595-020-02155-8.

2. Fränneby U, Gunnarsson U, Andersson M, Heuman R, Nordin P, Nyrén O, et al. Validation of an Inguinal Pain Questionnaire for 
assessment of chronic pain after groin hernia repair. Br J Surg. 2008;95:488-93.

3. Anthoine E, Moret L, Regnault A, Sébille V, Hardouin J-B. Sample size used to validate a scale: a review of publications on newly-developed patient reported outcomes measures. Health Qual Life Outcomes. 2014;12:2.

4. Olsson A, Sandblom G, Fränneby U, Sondén A, Gunnarsson U, Dahlstrand $U$. The short-form inguinal pain questionnaire (sfIPQ): an instrument for rating groin pain after inguinal hernia surgery in daily clinical practice. World J Surg. 2019;43:806-11.
5. Irarrázaval MJ, Inzunza M, Muñoz R, Quezada N, Brañes A, Gabrielli M, et al. Telemedicine for postoperative follow-up, virtual surgical clinics during COVID-19 pandemic. Surg Endosc. 2020. https://doi.org/10.1007/s00464-020-08130-1.

6. Keesara S, Jonas A, Schulman K, Massachusetts Medical Society. COVID-19 and health care's digital revolution. N Engl J Med. 2020. https://doi.org/10.1056/NEJMp2005835.

Publisher's Note Springer Nature remains neutral with regard to jurisdictional claims in published maps and institutional affiliations. 